Supplementary Information for:

\title{
Recyclable and Flexible Dual-Mode Electronics with Light and Heat Management
}

Tengyang Ye ${ }^{\dagger, \S}$ Fei Xiu, ${ }^{\dagger, \S}$ Shuai Cheng,,${ }^{\dagger} \S$ Chaoyi Ban, ${ }^{\dagger}$ Zhihui Tian, ${ }^{\dagger}$ Yingying Chen,${ }^{\dagger}$ Yamei Ding, ${ }^{\dagger}$ Ziwei Zhen, ${ }^{\dagger}$ Juqing Liu, ${ }^{*}, \dagger$ Wei Huang $*, \dagger$,

${ }^{\dagger}$ Key Laboratory of Flexible Electronics (KLOFE) and Institute of Advanced Materials (IAM), Nanjing Tech University (NanjingTech), 30 South Puzhu Road, Nanjing 211816, China

\$ Shaanxi Institute of Flexible Electronics (SIFE), Northwestern Polytechnical University (NPU), 127 West Youyi Road, Xi'an 710072, China

$\S$ These authors contributed equally.

*E-mail: iamjqliu@njtech.edu.cn

* E-mail: iamwhuang@ njtech.edu.cn 

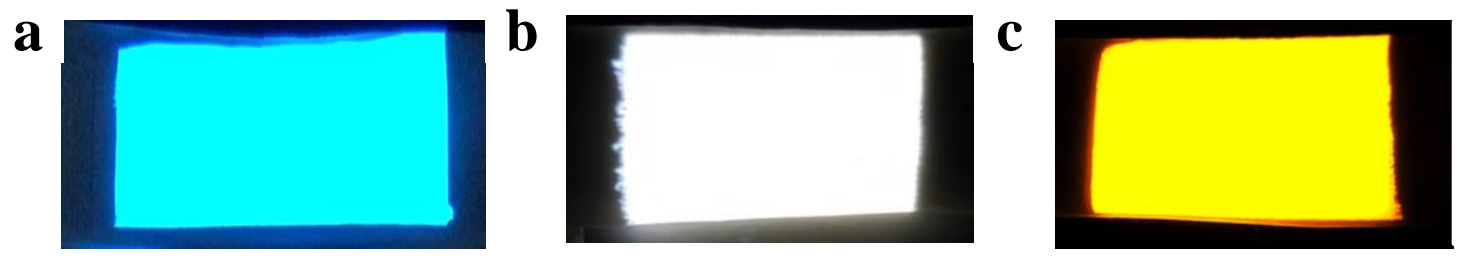

Figure S1. Photographs of the fabricated dual-mode device with two AgNW-PDMS electrodes. (a) Blue light emitting device. (b) White light emitting device. (c) Yellow light emitting device. 

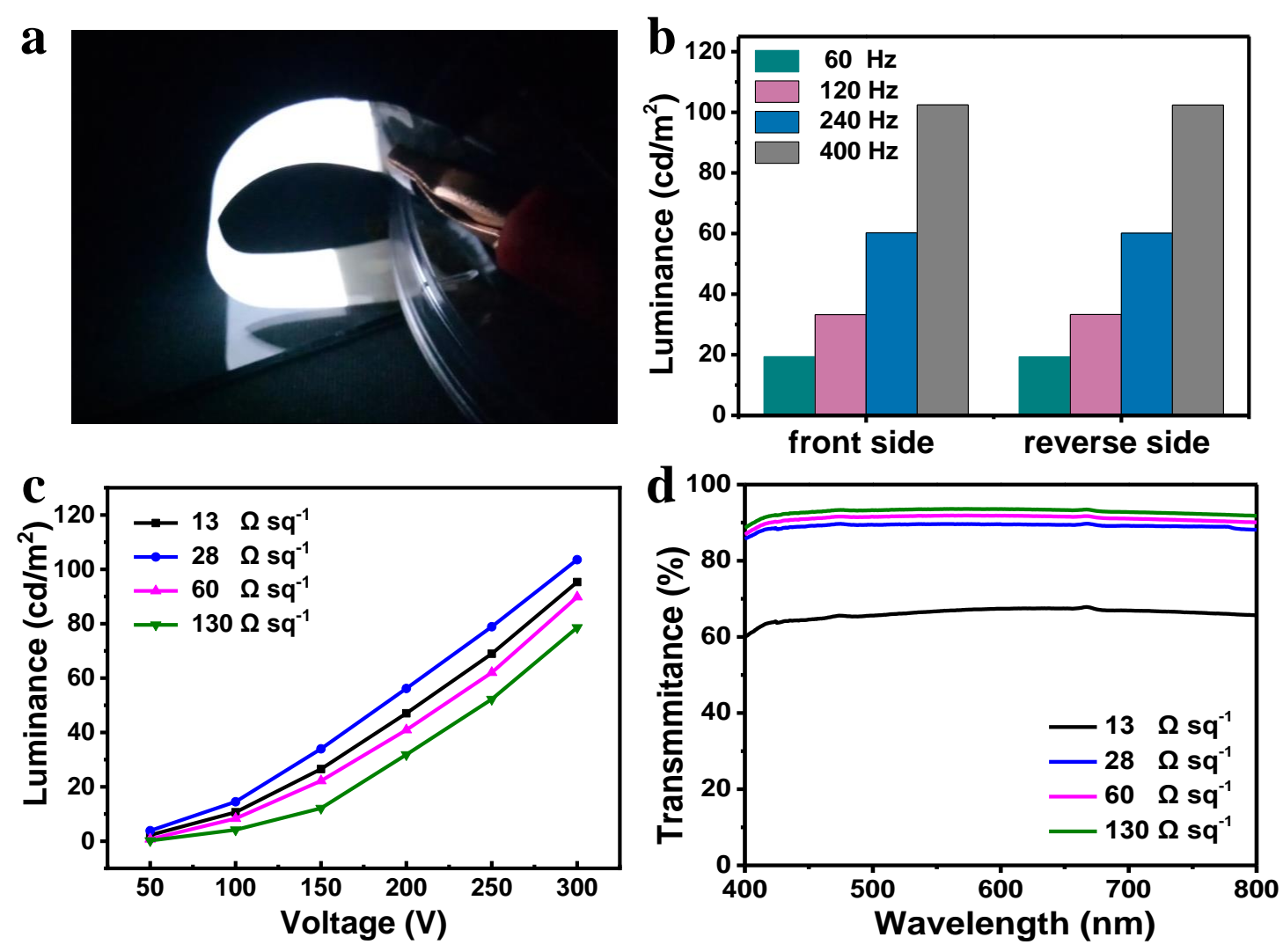

Figure S2. Photographs and luminance of dual-mode device. (a) Photographs of the fabricated electroluminescence device with great dual light emission because of the same transparent AgNW-NOA81 electrodes of both side with optical transmittance of 89\%. (b) The corresponding luminance is measured. (c) Luminance versus different sheet resistance of AgNW conductive networks. (d) Optical transmittance $(550 \mathrm{~nm})$ plotted versus sheet resistance.

Figure S2 shows that the dual-mode device exhibits good dual-side light emission with the electroluminescent intensity affected by sheet resistance of $\mathrm{AgNW}$ conductive networks and the best dual-side light emission is realized on the device with both transparent AgNW conductive networks with a sheet resistant of $28 \Omega \mathrm{sq}^{-1}$. 


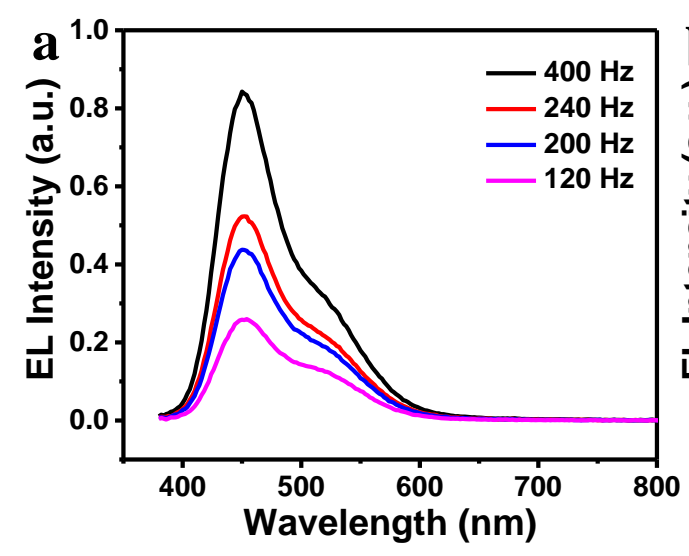

c

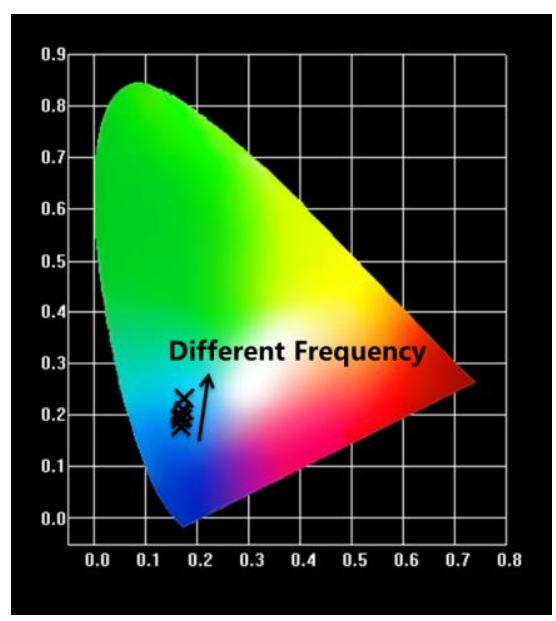

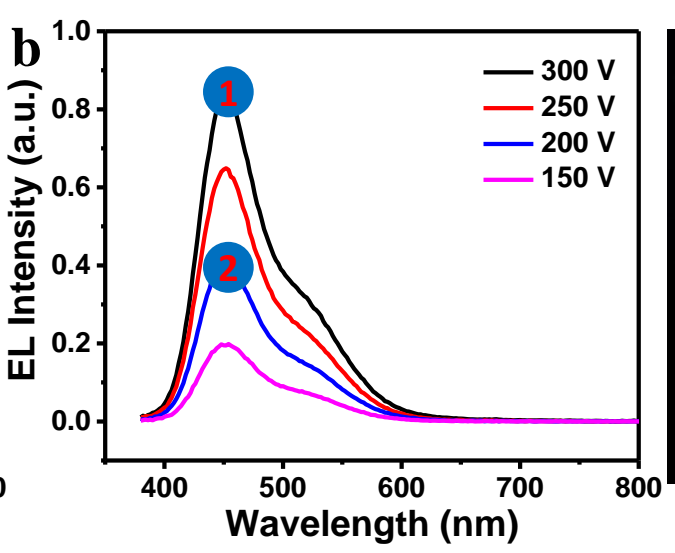

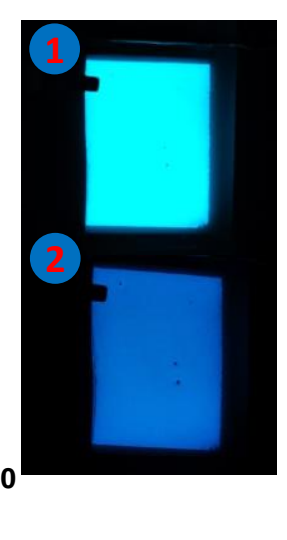

d

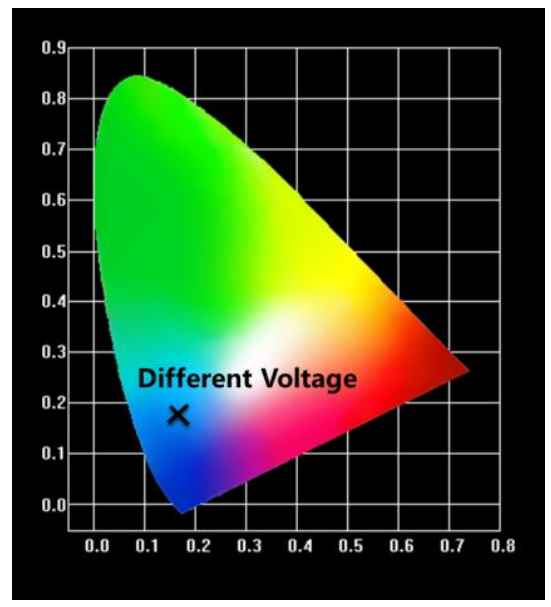

Figure S3. Optoelectronic characterization of the fabricated blue electroluminescence device. (a)

Electroluminescence emission spectra at different frequencies and (b) voltages. (c) (d) Relative CIE chromaticity diagram is shown. 


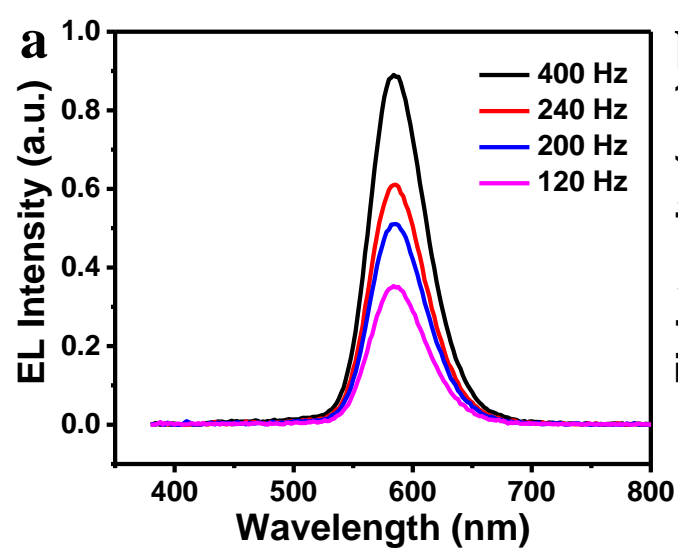

C

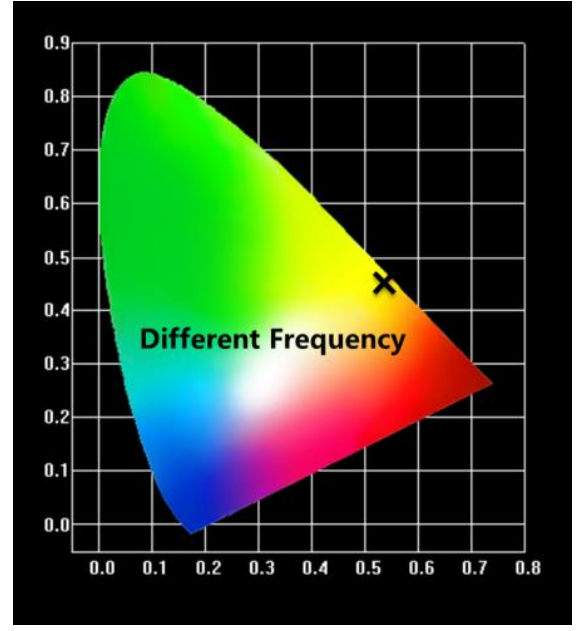

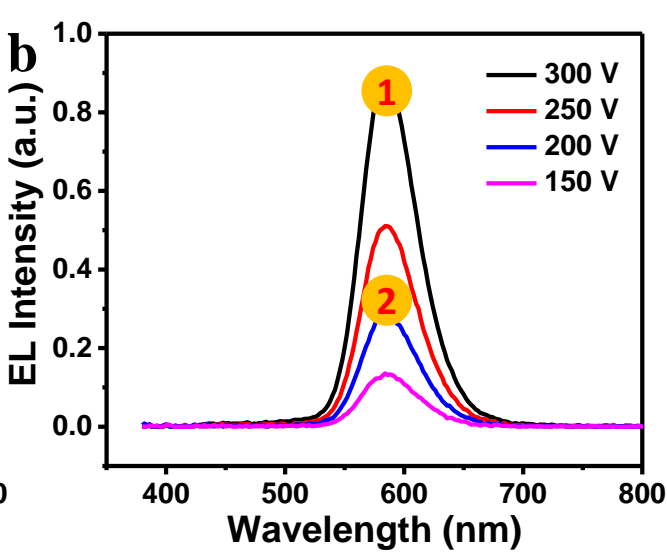
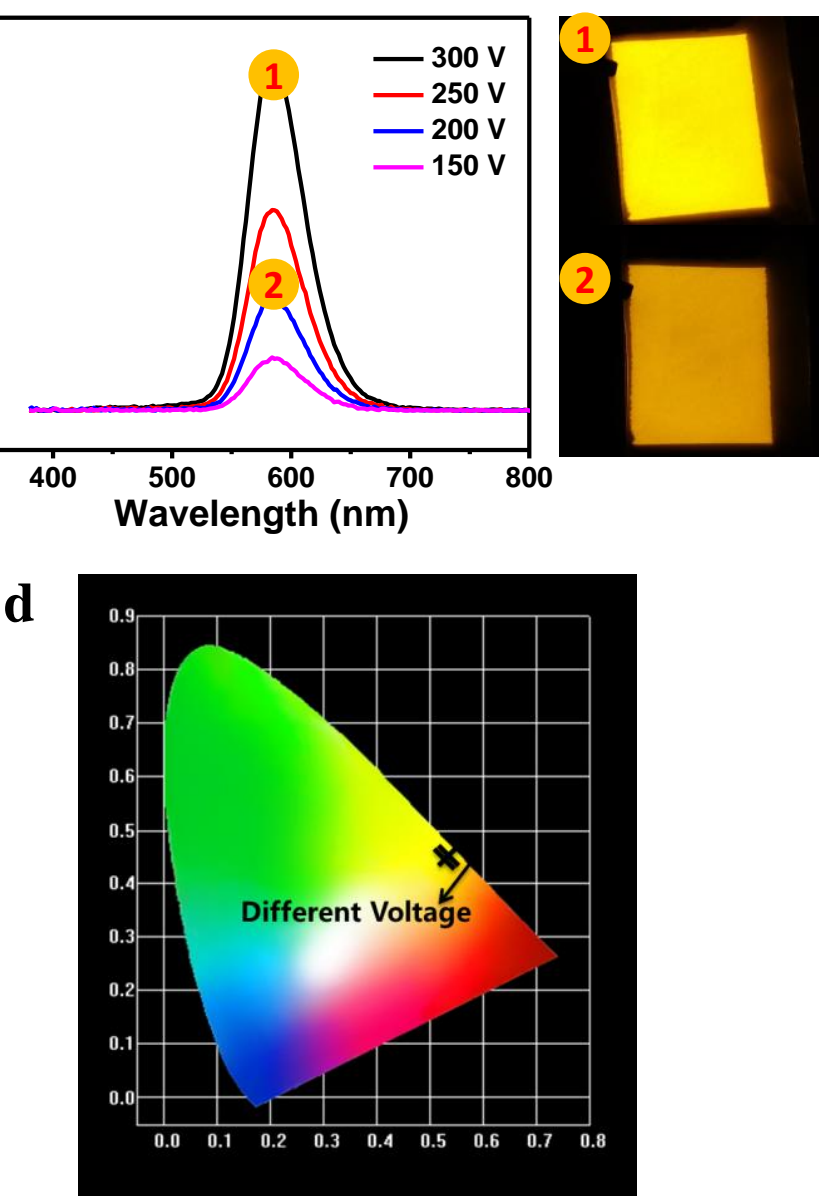

Figure S4. Optoelectronic characterization of the fabricated yellow electroluminescence device.

(a) Electroluminescence emission spectra at different frequencies and (b) voltages. (c) (d) Relative CIE chromaticity diagram is shown. 

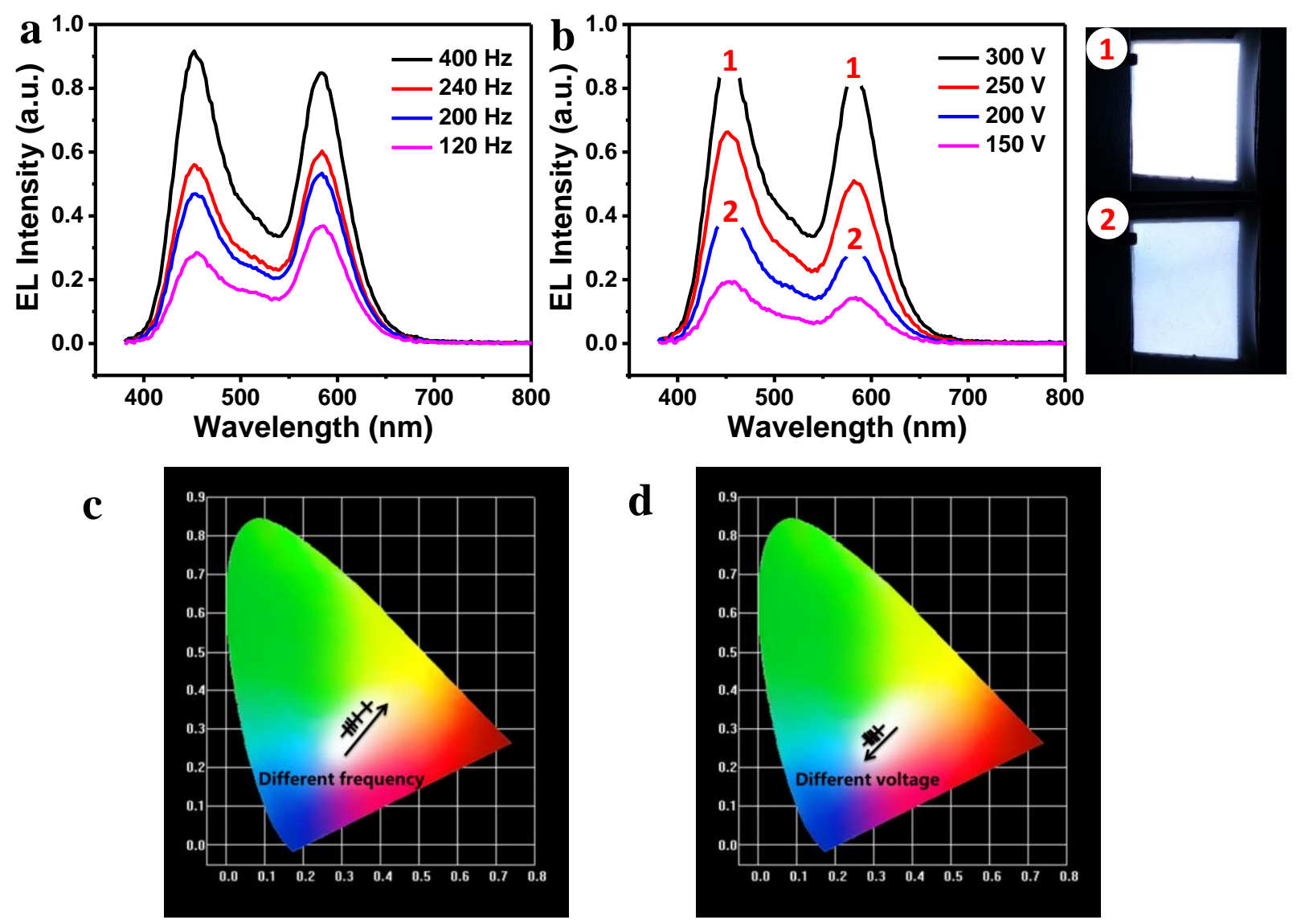

Figure S5. Optoelectronic characterization of the fabricated white electroluminescence device.

(a) Electroluminescence emission spectra at different frequencies and (b) voltages. (c) (d) Relative CIE chromaticity diagram is shown. 

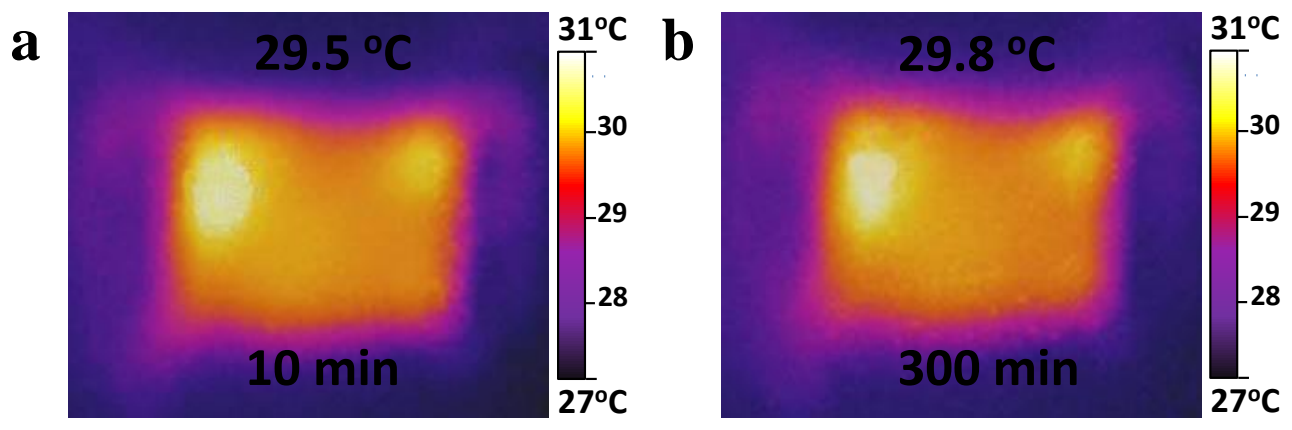

Figure S6. Temperature of the device at AC-200 V lighting for 300 min. (a) Infrared photographs of the electroluminescence device at $10 \mathrm{~min}$. (b) Infrared photographs of the electroluminescence device at $300 \mathrm{~min}$.

To give the heating performance data in the lighting mode under alternating current driving, temperature of the device is measured at AC-200 V lighting for $300 \mathrm{~min}$. It is observed that temperature of the device shows a slight increase in Figure S6, which stays consistent with the reported low power consumption of alternating current electroluminescent device (ACEL). ${ }^{1,2}$ This released heat is much smaller than that generated by the coplanar conductive film heating, so the temperature in the heating mode can be smart controlled via conductive film heating. 

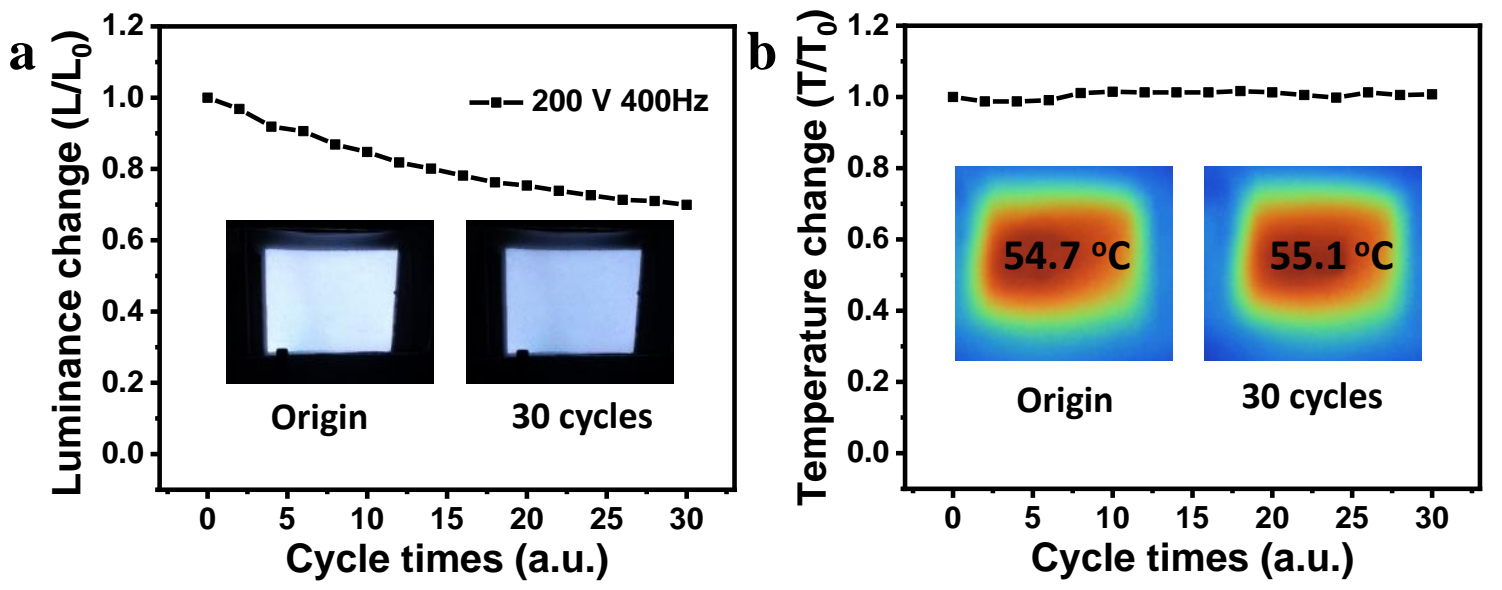

Figure S7. Stability of dual-mode thin-film device after 30 cycles. (a) Luminance change curves.

(b) Temperature change curves.

Stability of the dual-mode thin-film device is further investigated via performing the light mode for $10 \mathrm{~min}$ and heating mode work for $10 \mathrm{~min}$ as one cycle. The luminance showed a gradual decline trend in lighting mode after 30 cycles in Figure S7a, which gives rise to an exposure of the device to $\mathrm{H}_{2} \mathrm{O}$ and $\mathrm{O}_{2}{ }^{2,3}$ Furthermore, the temperature is found to be stable after 30 cycles benefited from the stability of AgNWs and the effective protection of its over-coating layers against oxidation and melting of AgNW network (Figure S7b). 

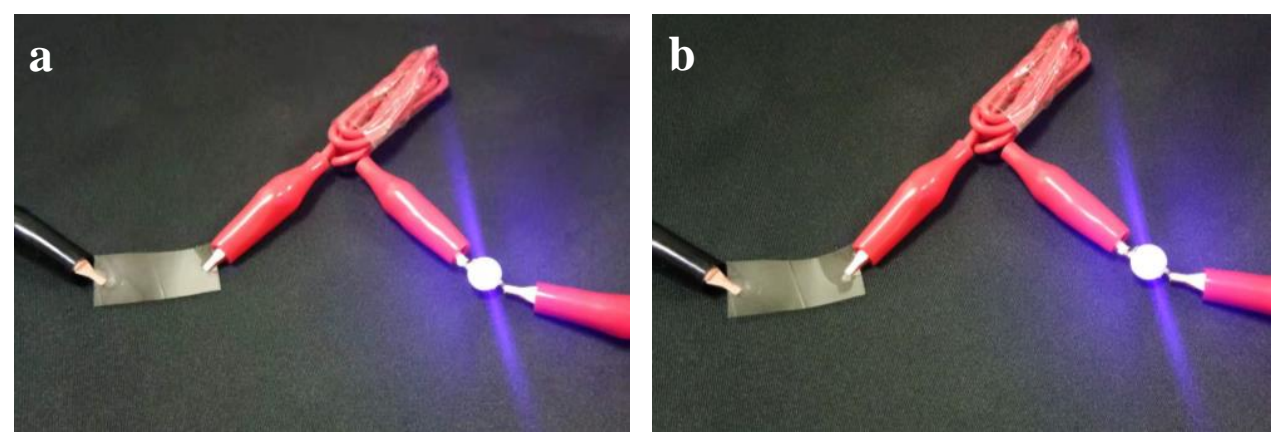

Figure S8. Photograph of LED illuminated by electrodes before (a) and after (b) water soaking. 

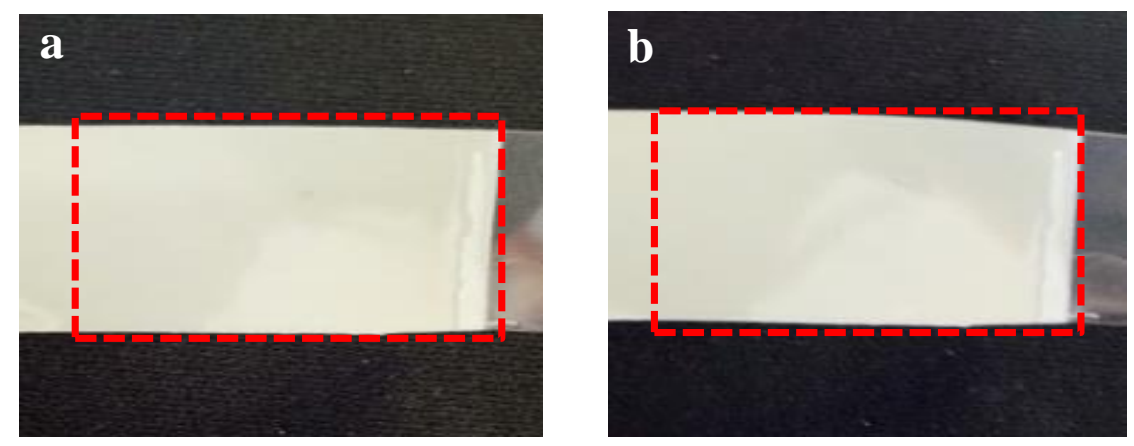

Figure S9. Photograph of the dual-mode lighting/heating device before (a) and after (b) recycling.

\section{REFERENCES}

1. Hu, B.; Li, D.; Ala, O.; Manandhar, P.; Fan, Q.; Kasilingam, D.; Calvert, P. D. Textile-Based Flexible Electroluminescent Devices. Adv. Funct. Mater. 2011, 21, 305-311.

2. Wood, V.; Halpert, J. E.; Panzer, M. J.; Bawendi, M. G.; Bulovic, V. Alternating Current Driven Electroluminescence from ZnSe/ZnS:Mn/ZnS Nanocrystals. Nano Lett. 2009, 9, 23672371.

3. Lee, S. W.; Cho, S. H.; Kang, H. S.; Kim, G.; Kim, J. S.; Jeong, B.; Kim, E. H.; Yu, S.; Hwang, I.; Han, H.; Park, T. H.; Jung, S. H.; Lee, J. K.; Shim, W.; Park, C. Electroluminescent Pressure-Sensing Displays. ACS Appl. Mater. Interfaces 2018, 10, 13757-13766. 